\title{
Prevalência de sobrepeso e obesidade em escolares: revisão sistemática de literatura
}

\section{The prevalence of overweight and obesity in schools: systematic literature review}

DOI: $10.46919 / \operatorname{archv} 1 \mathrm{n} 5-011$

Recebimento dos originais: 10/07/2020

Aceitação para publicação: 30/08/2020

\author{
Anderson Dos Santos Cantanhede \\ Especialista em ciência da saúde e do esporte e Docência do ensino superior \\ Instituição: Universidade Ceuma \\ Endereço: Av. São Luís Rei de França, 50 - Turu, São Luís - MA, 65065-470 \\ E-mail: anderson_cantanhede@hotmail.com
}

Eder Rodrigo Mariano

Doutorando em Educação

Instituição: Universidade Lusófona De Humanidades E Tecnologias

Endereço: Campo Grande 376, 1749-024 Lisboa, Portugal

E-mail: mariano_eder@hotmail.com

\section{RESUMO}

A obesidade é um dos problemas de saúde mais graves e crescentes que afeta a população mundial. Atualmente, é considera um problema de saúde pública, especialmente pelo fato do aumento de sua prevalência em crianças e adolescentes. No Brasil, percebem-se dados preocupantes em todas as regiões, fundamentalmente entre escolares. As causas da obesidade são de etiologia multifatorial, sendo as principais delas a inatividade física e a alimentação inadequada, rica em gorduras. O objetivo deste estudo foi analisar a prevalência de sobrepeso e obesidade em escolares de forma a traçar estratégias de prevenção a essa enfermidade. Tratou-se de uma pesquisa de revisão sistemática da literatura com escolares de 6 a 10 anos que teve como base de dados artigos e periódicos publicados eletronicamente nas bases de dados: Scielo, Pubmed, Lilacs e Biblioteca Virtual em Saúde no período compreendido entre janeiro de 2002 e janeiro de 2013. Estudos apontam que devido ao avanço tecnológico e a transição nutricional no Brasil as crianças tem se tornado mais vulnerável ao risco de sobrepeso e obesidade visto que vivem mais tempo usando o computador, assistindo televisão ou vídeo game, preferem alimentos ricos em gorduras e açúcares e realizam pouca atividade física. $\mathrm{O}$ estudo também revelou uma maior prevalência de sobrepeso e obesidade em meninos e nas escolas privadas. Com a obtenção destes resultados evidencia-se a importância de implantar programas educacionais, voltados especialmente à formação de hábitos alimentares mais saudáveis e a prática regular de atividades físicas, desde a infância.

Palavras-Chave: obesidade, sobrepeso, escolas, alimentação.

\begin{abstract}
Obesity is one of the most serious and growing health problems affecting the world population. Currently, it is considered a public health problem, especially due to the increasing in children and teenagers. In Brazil, worrying data can be seen in all regions, mainly among schoolchildren. The causes of obesity are from multifactorial etiology, the main ones being physical inactivity and inadequate diet, rich in fats. The aim of this study was to analyze the prevalence of overweight and obesity in schoolchildren in order to outline strategies for preventing this disease. It was a systematic literature review research with schoolchildren aged 6 to 10 years old, whose database included articles and journals published
\end{abstract}


electronically in the databases: Scielo, Pubmed, Lilacs and Virtual Health Library in the period between January 2002 and January 2013. Studies show that due to technological advancement and nutritional transition in Brazil, children have become more vulnerable to the risk of overweight and obesity as they live longer using the computer, watching television or playing video games, prefer foods rich in fats and sugars, and perform little physical activity. The study also revealed a higher prevalence of overweight and obesity in boys on private schools. With the achievement of these goals, the importance of implementing educational programs is evidenced, especially aimed at the formation of healthier eating habits and regular practice of physical activities, since childhood.

Keywords: obesity, overweight, schools, food.

\section{INTRODUÇÃO}

A obesidade é considerada, em países desenvolvidos, um importante problema de saúde pública, e pela Organização Mundial de Saúde (OMS), uma epidemia mundial (Brasil, 2010a). Essa epidemia tornou-se preocupante, pois tem repercutido em grande proporção na população infanto-juvenil aumentando o índice de morbidades.

O Comitê de especialistas da Força Tarefa Internacional para Obesidade (International Obesity Task Force, IOTF), reunido para determinar à medida que seria mais apropriada para avaliar sobrepeso e obesidade nas populações de crianças e adolescentes do mundo. Com base nos dados de seis diferentes populações de vários países, dentre eles o Brasil. Cole et al. (2000) descreveram os valores correspondentes ao IMC - Índice de Massa Corporal (peso/ altura ${ }^{2}$ em $25 \mathrm{Kg} / \mathrm{m}^{2}$ e $30 \mathrm{Kg} / \mathrm{m}^{2}$ para sobrepeso e obesidade respectivamente, para crianças a partir de 2 anos até os 20 anos de idade ( COLE et al., 2000).

De acordo com a Organização Mundial de Saúde (OMS), atualmente, uma em cada dez crianças em todo o mundo é obesa, o que representa cerca de cento e cinquenta e cinco milhões (OMS, 2013).

A IOTF (2000) estima que, a nível mundial, cerca de 150 milhões de crianças em idade escolar têm excesso de peso e aproximadamente 45 milhões destas são obesas. Sendo que a prevalências de obesidade tem aumentado nos países em desenvolvimento bem como se evidencia também o excesso de peso nas classes econômicas mais altas, os quais apontam o fator socioeconômico a essa realidade.

Estudos apontam a obesidade como um problema nutricional mais prevalente nos Estados Unidos, chegando a afetar um terço da população adulta e adolescente. Estimativas indicam que não havendo intervenção, a população americana chegará a 2035 com $90 \%$ dos indivíduos com excesso de peso (FRISBERG, 2004).

Nas últimas décadas a prevalência da obesidade tem aumentado nos países em desenvolvimento bem como se evidencia também o excesso de peso nas classes econômicas mais altas, os 
quais apontam o fator socioeconômico a essa realidade. Segundo Melo et al (2010), no Brasil, nos últimos 30 anos, o número de crianças com peso acima do normal triplicou, enquanto nos Estados Unidos duplicou. Esse aumento foi mais importante nas regiões Sul e Sudeste do Brasil, nas camadas mais favorecidas da população.

Ainda, segundo o Manual de Orientação da Obesidade na infância e adolescência (SBP, 2008), estudos realizados no Brasil apontam prevalências de excesso de peso que variam entre 10,8\% e 33,8\%, nas mais diferentes regiões do país e que abrangem a faixa etária infantil. Dados da Associação Brasileira para o Estudo da Obesidade, mostram que aproximadamente $40 \%$ da população brasileira apresentam excesso de peso e que a obesidade triplicou nos últimos vinte anos; já as crianças encontramse $15 \%$ acima do peso e $5 \%$ são obesas (ABESO, 2009).

As modificações nos padrões alimentares e de atividade física bem como o meio ambiente e o contexto familiar desfavoráveis tem propiciado condições ao desenvolvimento do excesso de peso e da obesidade na infância e na adolescência (WHO 2009c).

As transições econômicas, sociais, demográficas e relacionadas à saúde no Brasil a partir do século XX apontam mudanças nos padrões alimentares da população substituindo a escassez de alimentos pelo excesso. A inserção de uma dieta rica em gorduras (principalmente as de origem animal), açúcar e alimentos refinados, e reduzidos em carboidratos complexos e fibras têm contribuído para o aumento da obesidade (TORRES, FURUMOTO e ALVES, 2013).

Segundo Melo, Luft e Meyer (2004), a obesidade determina diversos distúrbios tanto na infância e adolescência quanto na idade adulta, sendo na infância o controle mais difícil, pois está relacionada à dependência dos pais e consequentemente à mudança de hábitos, além da falta de explicação e entendimento da criança quanto aos problemas relacionados com a obesidade.

A prevalência de outras doenças crônicas não transmissíveis relacionadas ao sobrepeso, e principalmente, à obesidade cresce no mundo todo, alertando a pesquisadores e profissionais da área de saúde às medidas de intervenção (ABESO, 2009).

Ciente das consequências dessa epidemia que se inicia na infância e oferece riscos à saúde na adolescência e fase adulta emerge, com certa urgência, a necessidade de se traçar estratégias de prevenção. Desta forma, o presente trabalho torna-se relevante por apontar o aumento dos índices de sobrepeso e obesidade na infância, assim como os problemas de saúde que tais condições acarretam a esses indivíduos.

Neste contexto, este esta revisão sistemática tem como objetivo apontar a prevalência de sobrepeso e obesidade em escolares de 6 a 10 anos. 


\section{MATERIAL E MÉTODOS}

Esse estudo constitui uma revisão sistemática realizada por meio de busca de artigos publicados entre janeiro de 2002 a janeiro de 2013 na base de dados eletrônicos Google Acadêmico Scientific Electronic Library Online Brasil (SCIELO), Literatura Latino-Americana e do Caribe em Ciências da Saúde (LILACS), PubMed (US National Libary of Medicine, Bethesda, MD) e Biblioteca Virtual em Saúde (BVS, Ministério da Saúde, Brasil) que abordaram a prevalência de sobrepeso e obesidade. Foram utilizadas as palavras-chave "obesidade", "sobrepeso", "crianças", "escolares", "prevalência", "6 a 10 anos", "desde, 2002”, "incidência", "tratamento", "atividade física”, “exercício físico", “desde 2013”. Por meio da leitura dos artigos encontrados, foram escolhidos os que continham informações de acordo com os propósitos da pesquisa.

Dos 61.600 artigos apresentados a partir do primeiro descritor, obtivemos um total de 89 artigos após digitarmos o último descritor. Destes artigos, apenas 13 atenderam aos critérios de inclusão, abaixo:

- Estudos de prevalência de sobrepeso, obesidade (excesso de peso, estado nutricional), e/ou associação entre sobrepeso e obesidade (excesso de peso) em escolares;

- Estudos com critérios diagnósticos para cálculo de medida que tinham o índice de massa corporal (IMC), utilizando-se como valores de corte estabelecidos por Cole et al e OMS sendo consideradas com sobrepeso as crianças com IMC para a idade maior ou igual ao percentil 85 e menor que o percentil 95; com obesidade, foram consideradas as crianças com o IMC para a idade maior ou igual ao percentil 95; os que usaram como padrão de referência as curvas de crescimento do National C'enter for Health Statistics (NCHS) de acordo com o sexo e a idade com base nas normas propostas pelo Center for Disease Control and Prevention (CDC), conforme a população de referência do National Health and Nutrition Examination Survey (NHANES I), como recomenda a OMS;

- Estudos publicados a partir de janeiro de 2002 a janeiro de 2013.

\section{RESULTADOS E DISCUSSÕES}

Inicialmente foram identificados 89 estudos, destes 13 abordaram o tema com o perfil da clientela de interesse. Das treze publicações analisadas, dez $(76,92 \%)$ abordaram a prevalência de sobrepeso e obesidade, enquanto que as demais descreveram de forma proporcionalmente iguais $(7,69 \%)$ a análise da obesidade e a prevalência da obesidade, sobrepeso, hábito alimentar e frequência de atividade física. 
Foram identificados dois tipos de escola, dentre as quais (6) - 46,15\% mostraram alunos de escolas públicas e privadas (5) - 38,46\% descreveram de escolas públicas e duas $(15,39 \%)$ somente alunos de escolas privadas.

Os resultados referentes à prevalência de sobrepeso e obesidade em todos os estudos avaliados e nas variáveis descritas são apresentados nas Tabelas 1 e 2. Esses resultados indicaram que, independente do gênero e do tipo de escola utilizados, a prevalência de sobrepeso e obesidade em escolares variaram de $5,6 \%$ a $25 \%$ e, $2,2 \%$ a $23 \%$, respectivamente.

Tabela 1 - Relação de estudos sobre prevalência de sobrepeso e obesidade em escolares de 6 a 10 anos, segundo o sexo (2002 a 2013).

\begin{tabular}{|c|c|c|c|c|c|c|c|c|c|}
\hline \multirow[t]{3}{*}{ Autor } & \multirow[t]{3}{*}{ Local } & \multirow{3}{*}{$\begin{array}{l}\text { Tipo de } \\
\text { escola }\end{array}$} & \multirow{3}{*}{$\begin{array}{l}\text { Faixa } \\
\text { etária }\end{array}$} & \multirow[t]{3}{*}{ Amostra } & \multirow{3}{*}{$\begin{array}{c}\text { Critério } \\
\text { Diagnóstico }\end{array}$} & \multicolumn{4}{|c|}{ Resultados } \\
\hline & & & & & & \multicolumn{2}{|c|}{ Sobrepeso } & \multicolumn{2}{|c|}{ Obesidade } \\
\hline & & & & & & Masc & Fem & Masc & Fem \\
\hline $\begin{array}{l}\text { FORTES, } \\
2003\end{array}$ & Florianópolis & $\begin{array}{c}\text { Publica } \\
\text { e } \\
\text { Privada }\end{array}$ & $\begin{array}{l}7 \text { a } 10 \\
\text { anos }\end{array}$ & 640 & IMC - IOFT & \multicolumn{4}{|c|}{$15,6 \%, 18,3 \% ; 12,2 \%, 4,9 \%$} \\
\hline $\begin{array}{l}\text { SOAR et al., } \\
2004\end{array}$ & Florianópolis & publica & $\begin{array}{l}7 \text { a } 9 \\
\text { anos }\end{array}$ & 419 & IMC & \multicolumn{2}{|c|}{$17,9 \%$} & \multicolumn{2}{|c|}{$6,7 \%$} \\
\hline $\begin{array}{c}\text { GIUGLIANO } \\
\text { E } \\
\text { CARNEIRO, } \\
2004\end{array}$ & $\begin{array}{l}\text { Taguatinga, } \\
\text { DF }\end{array}$ & Privada & $\begin{array}{l}6 \text { a } 10 \\
\text { anos }\end{array}$ & 452 & IMC - IOFT & \multicolumn{4}{|c|}{$16,7 \%, 16,9 \% ; 4,4 \%, 6,0 \%$} \\
\hline $\begin{array}{l}\text { RONQUE et } \\
\text { al,(2005) }\end{array}$ & $\begin{array}{l}\text { Londrina, } \\
\text { Paraná }\end{array}$ & Privada & $\begin{array}{l}7 \text { a } 10 \\
\text { anos }\end{array}$ & 511 & IMC - IOFT & \multicolumn{4}{|c|}{$19,7 \%, 17,3 \% ; 17,5 \%, 9,3 \%$} \\
\hline $\begin{array}{c}\text { COSTA, } \\
\text { CINTRA } \\
\& \text { FISBERG, } \\
2006\end{array}$ & Santos - SP & $\begin{array}{l}\text { Publica } \\
\text { e } \\
\text { Privada }\end{array}$ & $\begin{array}{l}7 \text { a } 10 \\
\text { anos }\end{array}$ & 10.822 & $\begin{array}{l}\text { IMC (Cole } \\
\text { et al) }\end{array}$ & \multicolumn{4}{|c|}{$13,7 \%, 14,8 \% ; 16,9 \%, 14,3 \%$} \\
\hline $\begin{array}{c}\text { CASANOVA, } \\
2007\end{array}$ & $\begin{array}{c}\text { Balneário } \\
\text { Camboriú, SC }\end{array}$ & publica & $\begin{array}{l}6 \text { a } 10 \\
\text { anos }\end{array}$ & 624 & IMC - IOFT & \multicolumn{4}{|c|}{$5,6 \%, 6,9 \% ; 3,9 \%, 4,8 \%$} \\
\hline $\begin{array}{l}\text { RICARDO et } \\
\text { al. } 2009\end{array}$ & $\begin{array}{c}\text { Santa } \\
\text { Catarina }\end{array}$ & $\begin{array}{c}\text { Publica } \\
\text { e } \\
\text { Privada }\end{array}$ & $\begin{array}{l}6 \text { a } 10 \\
\text { anos }\end{array}$ & 4.964 & IMC - IOFT & \multicolumn{4}{|c|}{$14,9 \%, 15,9 \% ; 6,7 \%, 5,5 \%$} \\
\hline $\begin{array}{l}\text { NETTO- } \\
\text { OLIVEIRA et } \\
\text { al. } 2010\end{array}$ & $\begin{array}{l}\text { Maringá, } \\
\text { Paraná }\end{array}$ & $\begin{array}{l}\text { publica } \\
\text { e } \\
\text { privada }\end{array}$ & $\begin{array}{c}6,0 \text { a } \\
7,9 \\
\text { anos }\end{array}$ & 1634 & $\begin{array}{l}\text { IMC (Cole } \\
\quad \text { et al) }\end{array}$ & \multicolumn{4}{|c|}{$6,7 \%, 7,1 \% ; 4,3 \%, 4,0 \%$} \\
\hline $\begin{array}{c}\text { MELO et al. } \\
2010\end{array}$ & $\begin{array}{l}\text { Marialva - } \\
\text { Paraná }\end{array}$ & Publica & $\begin{array}{l}6 \text { a } 10 \\
\text { anos }\end{array}$ & 356 & $\begin{array}{l}\text { IMC (Cole } \\
\text { et al - IOTF }\end{array}$ & \multicolumn{4}{|c|}{$20 \%$} \\
\hline $\begin{array}{l}\text { Medeiros et } \\
\text { al, } 2011\end{array}$ & $\begin{array}{l}\text { Campina } \\
\text { Grande, } \\
\text { Paraíba }\end{array}$ & $\begin{array}{c}\text { publica } \\
\mathrm{e} \\
\text { 1privada }\end{array}$ & $\begin{array}{l}6 \text { a } 10 \\
\text { anos }\end{array}$ & 285 & $\begin{array}{l}\text { IMC (Cole } \\
\text { et al) }\end{array}$ & \multicolumn{2}{|r|}{$14,7 \%$} & \multicolumn{2}{|l|}{$8,8 \%$} \\
\hline $\begin{array}{l}\text { LACERDA E } \\
\text { MELO, } 2011\end{array}$ & $\begin{array}{l}\text { Taguatinga } \\
\text { Sul - DF }\end{array}$ & Pública & $\begin{array}{l}6 \text { a } 10 \\
\text { anos }\end{array}$ & 83 & $\begin{array}{l}\text { IMC (Cole } \\
\text { et al) }\end{array}$ & \multicolumn{4}{|c|}{$15,6 \%, 13,2 \% ; 2,2 \%, 2,6 \%$} \\
\hline SILVA, 2005 & $\begin{array}{l}\text { Fernandópolis } \\
-\mathrm{SP}\end{array}$ & $\begin{array}{l}\text { publica } \\
\text { e } \\
\text { privada }\end{array}$ & $\begin{array}{l}6 \text { a } 10 \\
\text { anos }\end{array}$ & 319 & $\begin{array}{l}\text { IMC (Cole } \\
\text { et al) }\end{array}$ & \multicolumn{4}{|c|}{$\begin{array}{l}{ }^{*} 22 \%, 25 \% ; 22 \%, 20 \% . \\
{ }^{* *} 18 \%, 19 \% ; 23 \%, 19 \%\end{array}$} \\
\hline $\begin{array}{l}\text { HONÓRIO e } \\
\text { HADLER, } \\
2012\end{array}$ & Goiânia - GO & Publica & $\begin{array}{l}6 \text { a } 9 \\
\text { anos }\end{array}$ & 320 & $\begin{array}{c}\mathrm{IMC} \\
(\mathrm{CDC})\end{array}$ & \multicolumn{4}{|c|}{$23,6 \%, 18,2 \% ; 10,6 \% ; 5,6 \%$} \\
\hline
\end{tabular}

Legenda: *Escola Pública; ${ }^{* *}$ Escola Privada 
Com relação à prevalência de sobrepeso e obesidade associados entre meninos e meninas. Fortes (2003) avaliou que os meninos apresentaram uma maior prevalência de obesidade do que as meninas, independentemente do tipo de escola.

Silva, 2005; Netto-Oliveira et al. 2010; Medeiros et al, 2011 e Costa, Cintra \&Fisberg, 2006, compararam a prevalência de obesidade e sobrepeso em escolas privadas e públicas, e perceberam uma porcentagem maior de crianças com excesso de peso ainda nas camadas mais ricas da sociedade, representadas pelas escolas particulares. No entanto, o risco para sobrepeso foi considerado maior nos alunos da escola pública, sugerindo que a situação nas camadas mais baixas da sociedade caminha nesta mesma direção (SILVA, 2005).

Quando essa prevalência foi associada às condições demográficas e socioeconômica dos escolares constatou-se que uma maior renda (Casa Nova, 2007; Ronque et al, 2005; Netto-Oliveira et al. 2010), estudar em escolas particulares (Costa, Cintra \& Fisberg, 2006; Fortes, 2003; ) pode influenciar o sobrepeso e a obesidade na criança. Esses autores verificaram que escolares de nível econômico mais privilegiado demonstraram risco, aproximadamente, duas vezes maior de apresentar excesso de peso em relação aos de nível mais baixo. A ocorrência de sobrepeso e obesidade em crianças foi associada também à baixa escolaridade materna (GIUGLIANO e CARNEIRO, 2004).

Dessa forma, Ronque et al. (2005) verificaram a prevalência de sobrepeso e obesidade em escolares brasileiros na faixa etária entre sete e dez anos, de ambos os sexos e de alto nível socioeconômico, e os resultados indicam taxas de sobrepeso $(19,7 \%)$ e de obesidade $(17,5 \%)$, ambos em escolares do sexo masculino (Tabela1). Estes autores também estabeleceram a relação da obesidade nas duas fases da vida e estimaram que crianças obesas podem apresentar entre $68 \%$ e $77 \%$ de probabilidade de permanecerem obesas na adolescência, enquanto que adolescentes obesos podem ter risco de $30 \%$ a $50 \%$ de obesidade na idade adulta.

Soar et al. (2004), sugeriram que a circunferência da cintura (CC) seja utilizada juntamente com o IMC no diagnóstico do tipo de adiposidade. Este estudo avaliou 419 escolares de 7 a 9 anos e verificou que a circunferência da cintura apresentou boa correlação com o IMC (Tabela1). Estes afirmaram que além de conhecer o grau de obesidade, também se torna importante conhecer a distribuição da gordura corporal visto ser a alteração desta medida um preditor de risco para as síndromes metabólicas em crianças e adolescentes (diabetes mellitus tipo 2, hipertensão arterial e dislipidemias). Segundo os autores os resultados são preocupantes, pois o excesso de adiposidade central é associado com riscos de doenças cardiovasculares em crianças, adolescentes e adultos (SBP, 2008). 
Tabela 2 - Relação de estudos sobre prevalência de sobrepeso e obesidade em escolares de 6 a 10 anos, segundo o tipo de escola e nível socioeconômico (2002 a 2013).

\begin{tabular}{|c|c|c|c|c|c|c|}
\hline Autor & Local & $\begin{array}{l}\text { Tipo de } \\
\text { escola }\end{array}$ & $\begin{array}{l}\text { Faixa } \\
\text { etária }\end{array}$ & Amostra & $\begin{array}{c}\text { Critério } \\
\text { Diagnóstico }\end{array}$ & Resultados \\
\hline $\begin{array}{c}\text { FORTES, } \\
2003\end{array}$ & Florianópolis & $\begin{array}{l}\text { Publica } \\
\text { e } \\
\text { Privada }\end{array}$ & $\begin{array}{l}7 \text { a } 10 \\
\text { anos }\end{array}$ & 640 & IMC - IOFT & $\begin{array}{c}\text { SB: M } 15,6 \% \text { F- } \\
18,3 \% \text {; } \\
\text { OB: M: } 12,2 \%, F- \\
4,9 \%\end{array}$ \\
\hline $\begin{array}{c}\text { Costa, } \\
\text { Cintra } \\
\text { \&Fisberg, } \\
2006\end{array}$ & Santos - SP & $\begin{array}{l}\text { Publica } \\
\text { e } \\
\text { Privada }\end{array}$ & $\begin{array}{l}7 \text { a } 10 \\
\text { anos }\end{array}$ & 10.822 & $\begin{array}{c}\text { IMC (Cole et } \\
\text { al) }\end{array}$ & $\begin{array}{c}\text { SB M- } 13,7 \% ; \text { F- } \\
\text { 14,8\% EPB; } \\
\text { 17,7\% nos meninos e } \\
22,2(\mathrm{EPV}) \\
\text { OB- } 16,9 \% \text { dos } \\
\text { meninos e } 14,3 \%( \\
\text { EPB); } 29,8 \% \text { dos } \\
\text { meninos e } 20,3 \%\end{array}$ \\
\hline $\begin{array}{l}\text { SILVA, } \\
2005\end{array}$ & $\begin{array}{c}\text { Fernandópolis } \\
-\mathrm{SP}\end{array}$ & $\begin{array}{l}\text { Publica } \\
\text { e } \\
\text { Privada }\end{array}$ & $\begin{array}{c}6 \text { a } 10 \\
\text { anos }\end{array}$ & 319 & $\begin{array}{c}\text { IMC (Cole et } \\
\text { al }\end{array}$ & $\begin{array}{l}\text { SP EPB M: } 22 \% ; \mathrm{F}: \\
25 \% \\
\text { EPV- M: } 18 \% \text {; F: } 19 \% \\
\text { OB- EPB M: } 22 \% ; \mathrm{F}: \\
20 \% \text {. } \\
\text { EPV- M: } 23 \%, \mathrm{~F}: 19 \%\end{array}$ \\
\hline $\begin{array}{l}\text { Medeiros et } \\
\text { al, 2011 }\end{array}$ & $\begin{array}{l}\text { Campina } \\
\text { Grande, } \\
\text { Paraíba }\end{array}$ & $\begin{array}{l}\text { publica } \\
\text { e } \\
\text { privada }\end{array}$ & $\begin{array}{c}6 \text { a } 10 \\
\text { anos }\end{array}$ & 255 & $\begin{array}{l}\text { IMC (Cole et } \\
\text { al) }\end{array}$ & $\begin{array}{c}\mathrm{SP}-14,7 \% \\
\mathrm{OB}-8,8 \%\end{array}$ \\
\hline $\begin{array}{c}\text { Lacerda e } \\
\text { Melo, } 2011\end{array}$ & $\begin{array}{c}\text { Taguatinga Sul } \\
- \text { DF }\end{array}$ & Pública & $\begin{array}{c}6 \text { a } 10 \\
\text { anos }\end{array}$ & 83 & $\begin{array}{l}\text { IMC (Cole et } \\
\text { al) }\end{array}$ & $\begin{array}{l}\text { Sobrepeso: } \mathrm{M}-15,6 \% \\
\text { e F-13,2\%; O: M - } \\
2,2 \%, \mathrm{~F}-2,6 \% \text {; Nível } \\
\text { sócio econ: SP (classes } \\
\text { A, B, C e D) - } 9,6 \% \text {; O } \\
\text { (D e E) - } 2,4 \%\end{array}$ \\
\hline
\end{tabular}

Legenda: SB - sobrepeso; OB - obesidade; M - masculino; F - feminino; EPB - escola pública; EPV - escola privada

Ao analisar as condições de sedentarismo, dieta e atividade física, Fortes (2003) observou-se também que os fatores que se ajustaram ao modelo logístico multivariado para obesidade, a respeito da criança estar ou não fazendo dieta, foi o sexo masculino, o sobrepeso e/ou obesidade dos pais, consumo de lanches com alto teor de gordura de 4 a 7 vezes por semana e passar mais de 4 horas por dia assistindo TV. Silva (2010) verificou em seu estudo que 59\% dos escolares preferiam jogar videogame, ver TV ou computador. Nessa mesma análise, Casanova (2007) observou que 90,2\% dos escolares divertiam-se em computador e videogame em média entre 0 a 2 horas/dia bem como crianças que assistem mais televisão comem menos verduras, legumes e consumem doces no café da manhã. Esses hábitos estão associados à maior prevalência de sobrepeso e obesidade. Medeiros (2011) descreveu que 66,3\% dos alunos praticavam menos que três dias de atividade física por semana. Melo (2010) avaliou que 51,6\% dos meninos dispendiam maior número de horas em atividades sedentárias.

De acordo com Alves et al. (2005) podem ser elencados também fatores de risco para o sedentarismo, tais como pais fisicamente inativos, escolas sem atividades esportivas, ser do sexo feminino, residir em área urbana e presença de televisão no quarto da criança. 
A causa primária da obesidade é o desequilibro crônico entre a ingestão alimentar e o gasto energético, o que resulta de elevado consumo calórico e pouca atividade física (MEIRELLES; GOMES, 2004). Disso se depreende que a combinação de dieta e exercícios pode proporcionar perda de peso mais eficiente durante curto ou longo prazo, em comparação à apenas uma dessas intervenções isoladamente (HAUSER et al., 2004).

As crianças estão mais sedentárias. Houve aumento de tempo de tela (TV, computador, videogames) e redução das atividades físicas. Existem evidências científicas demostrando que a atividade sedentária se correlaciona com a obesidade infantil, que os obesos são menos ativos e queimam menos calorias e ainda que, à medida que progridem da infância para a adolescência, as atividades sedentárias aumentam (JANZ, 2005).

De acordo com Mondini e Colaboradores (2007), a prevalência de crianças ingressantes na primeira série do ensino fundamental, em uma região metropolitana de São Paulo, que apresentaram sobrepeso foi de $10,8 \%$ e obesidade foi de 6,2\%. Foram associados a'mães obesas', elevado consumo de alimentos "não saudáveis", assistirem a televisão diariamente por quatro horas ou mais e a disponibilidade domiciliar per capta de óleo acima da recomendada.

De acordo com o Instituto Brasileiro de Geografia e Estatística quase 90\% das crianças brasileiras consomem gordura acima do recomendado, enquanto o excesso de açúcar faz parte da dieta de $80 \%$ delas (BRASIL, 2007).

\section{PROPOSTAS DE ATUAÇÃO PARA MUDANÇA DO QUADRO DE OBESIDADE INFANTIL}

A importância de prevenir a obesidade na infância decorre de sua associação com doenças crônicas não transmissíveis no adulto, que podem se instalar desde a infância.

Os estudos mostram os fatores ambientais como um dos principais vilões para ascensão da obesidade infantil. Estes fatores caracterizam-se pela alimentação desequilibrada e a falta de exercícios físicos mediados pelo sedentarismo. Frente a tal problema a qual enfrenta a população mundial torna-se necessário ocorrer mudança na alimentação e alteração no estilo de vida sedentário. Porém, para que isso aconteça, é imprescindível a participação ativa da família, dos professores e da comunidade escolar, já que as crianças tendem a reproduzir as práticas de seus familiares e educadores (SILVA, 2005; MEDEIROS, 2011 e MELO, 2010)

Estudos evidenciaram a atividade física como fator coadjuvante de combate e prevenção da obesidade infantil. O exercício físico contribui para o afastamento da criança da televisão, dos computadores e dos jogos de videogame, visto que essas práticas sedentárias têm uma grande contribuição no crescimento da obesidade infantil. Nota-se que são nessas atividades que as crianças 
tendem a consumir alimentos ricos em gorduras e açúcares e de baixo valor nutricional (SILVA, 2005; GIUGLIANO E CARNEIRO, 2004).

A pactuação entre a família e a escola a essa estratégia de prevenção torna-se primordial de uma vez que a alimentação dos filhos, por vezes, é influenciada pelos hábitos dos pais, em contrapartida, é na escola que as crianças despertam para as normas educacionais. Devem-se implantar estratégias de prevenção sistemática e de fácil aceitação com participação de uma equipe multidisciplinar mediado primeiramente, com propostas de sensibilização e, por fim, conscientização da clientela cuja meta será envolver a população às condições de vida saudáveis com início desde a infância. Afinal, trabalhar a qualidade de vida das crianças é diminuir as taxas de morbidade e mortalidade ainda precocemente.

De acordo com Costa et al (2001) a Educação Física auxilia na conscientização das crianças em fazer atividade física no dia a dia e também fazer com que esses alunos pensem mais na sua qualidade de vida, melhorando seus hábitos alimentares.

Brasil (2006) discorre que a escola é o ambiente mais propício para a implantação de hábitos saudáveis na prevenção da obesidade infantil em virtude de ser ela a formadora social e intelectual da população.

A Organização Pan-Americana da Saúde recomenda manter atividades físicas diárias, consumir mais frutas e verduras, trocar gorduras saturadas de origem animal por gorduras insaturadas de origem vegetal e diminuir a quantidade de alimentos gordurosos da dieta alimentar (OPAS, 2003).

A finalidade e importância da Educação Física Escolar como colaboradora na prevenção da obesidade infantil é um aspecto abordado hoje pelas ciências e importante que se perceba a relevância das interferências positivas que o profissional de Educação Física pode exercer sobre a formação de hábitos saudáveis na infância refletindo positivamente na vida adulta. (MAESTRI; FIAMONCINI, 2006).

\section{CONCLUSÃO}

Este estudo revelou que a maioria dos autores apontou que nas escolas particulares a prevalência de obesidade foi maior do que nas escolas públicas, entretanto, o sobrepeso foi evidenciado com mais frequência em escolas públicas demostrando dessa forma que as camadas mais baixas da sociedade já trilham passos para a obesidade.

Pode-se observar que dentre os principais fatores que influenciam a gênese da obesidade relaciona-se o ambiente devido às mudanças comportamentais como os maus hábitos alimentares e o sedentarismo. Tais comportamentos em crianças mostraram-se preocupante pelo fato destas passarem maior parte do dia assistindo televisão ou jogando vídeo-game em detrimento de brincadeiras mais ativas. 
Consequentemente, estas, consumem alimentos ricos em açúcares simples e gordura, com alta densidade energética e gastam menos energia.

Os estudos apontam a necessidade de medidas de intervenção, particularmente, em nível escolar como alternativa para prevenir ou minimizar o problema da obesidade. Frente a tal epidemia torna-se prudente ser a escola um local privilegiado de intervenção, onde pequenas mudanças, ao nível da alimentação e atividade física, podem contribuir para travar a escalada da obesidade e conduzir a estilos de vida mais saudáveis. Todavia, devem ser inseridos nas políticas de prevenção a tal epidemia uma equipe multidisciplinar a fim de inserir em tais intervenções, não somente os escolares, mas também como a sua família visto ser a educação a melhor forma de se propiciar a saúde.

\section{REFERÊNCIAS}

ABRAnTES, M. M.; LAMOUNIER, J. A., COLOSIMO, E. A. Prevalência de Sobrepeso e Obesidade nas Regiões Nordeste e Sudeste do Brasil Rev Assoc Med Bras; 49(2): 162-6, 2003

ALVES, J. G. B.; MONTENEGRO, F. M. U.; OLIVEIRA, F.A.; ALVES, R. V. Prática de esportes durante a adolescência e atividade física de lazer na vida adulta. Revista Brasileira de Medicina do Esporte, Niterói, v.11, n.5, p.291-4, 2005. http://dx.doi.org/10.1590/S1517-86922005000500009

AMERICAN ACADEMY OF PEDIATRICS (AAP). Prevention of Pediatric Overweight and Obesity. The Journal of Pediatrics. 2003; 112(2): 424-430

ABESO .Associação Brasileira para o Estudo da Obesidade e da Síndrome Metabólica. Diretrizes Brasileiras de Obesidade 2009/2010. 3.ed. - Itapevi, SP : AC Farmacêutica, 2009.

BRASIL. Lei no. 12.061, de 18 de dezembro de 2001. Dispõe sobre critérios de concessão de serviços de lanches e bebidas nas unidades educacionais, localizadas no Estado de Santa Catarina. Brasília (DF): Diário Oficial do Estado de Santa Catarina, 2001.

Lei no. 3.695, de 8 de novembro de 2005. Dispõe sobre a promoção da alimentação saudável nas escolas da rede de ensino do Distrito Federal. Brasília (DF): Diário Oficial do Distrito Federal, 2005.

Ministério da Saúde. Experiências estaduais e municipais de regulamentação da comercialização de alimentos em escolas no Brasil: identificação e sistematização do processo de construção e dispositivos legais adotados. Brasília (DF): Ministério da Saúde, 2007.

Parâmetros curriculares nacionais: educação física / Ministério da Educação. Secretaria da Educação Fundamental. $3^{\mathrm{a}}$ ed. Brasília: A Secretaria, 2007.

CASANOVA, Mariene. PREVALÊNCIA DE SOBREPESO INCLUINDO OBESIDADE EM ESCOLARES ENTRE 6 A 10 ANOS DE IDADE MATRICULADOS NO ENSINO PÚBLICO MUNICIPAL DE BALNEÁRIO CAMBORIÚ, SANTA CATARINA, BRASIL. Revista de Nutrição, Campinas, SP, v.26, n.4, p.103-11, 2007.

CAMPOS, Fabiano S. et al. PREVALÊNCIA DE OBESIDADE INFANTIL EM ALUNOS DO $5^{\circ}$ ANO DO ENSINO FUNDAMENTAL. Anais da Semana Educa, v. 1, n. 1, 2011. 
COSTA, Roberto Fernandes da. CINTRA, Isa de Pádua e FISBERG, Mauro. Sobrepeso e Obesidade em Escolares de Santos. Arq Bras Endocrino e Metab. vol 50 nº 1 Fevereiro 2006

COSTA, Estér de Queirós; RIBEIRO, Victoria Maria Brant; RIBEIRO, Eliana Claudia de Otero. Programa de alimentação escolar: espaço de aprendizagem e produção de conhecimento. Revista de Nutrição, v. 14, n. 3, p. 225-229, 2001

CHEN X, et al. Is sleep duration associated with childhood obesity? A systematic review and metaanalysis. Obesity 2008; 16: 265-274.gain in infancy. Int J Epidemiol 2007; 36: 104-107.

DEMERATH EW, Choh AC, Czerwinski SA, et al. Genetic and environmental influences on infant weight and weight change: the Fels Longitudinal Study. Am J Hum Biol 2007; 19: 692-702.

FORTE, Cézar Figueiredo. PREVALÊNCIA DE SOBREPESO E OBESIDADE EM CRIANÇAS DE 7 A 10 ANOS DE IDADE, EM FLORIANÓPOLIS. Cadernos de Saúde Pública. 2003; 16 (3) 13201328.

International Obesity Task Force. Chilhood Obesity Report [Online]. 2004 [cited 2013 set. 21]; Available from: URL: http://www.iotf.org/popout.asp?linkto=http://www.iotf.org/media/IOTFmay28.pdf

HAUSER, C.; BENETTI, M.; REBELO, F. P. V. Estratégias para o emagrecimento. Revista Brasileira de Cineantropometria e Desempenho Humano $=$ Brazilian Journal of Kinanthropometry and Human Performance, v.6, n.1, p.72-81, 2004. Disponível em: http://www.rbcdhonline. ufsc.br/viewarticle.php?id=96\&layout=abstract Acesso em: 13 out. 2013

KAVEY, R-E, W; et al. American heart association guidelines for primary prevention

of atherosclerotic cardiovascular disease beginning in childhood. The Journal of

Pediatrics. 2003; 142: 368-372.

HONÓRIO, Renata Félix e HADLER, Maria Claret Costa Monteiro. PREVALÊNCIA DE OBESIDADE E SOBREPESO EM CRIANÇAS DO PROGRAMA SAÚDE NA ESCOLA DE GOIÂNIA-GO. Cadernos de Saúde Pública. 2012; 29(13):1309-1316.

JANZ KF, Burns TL, Levy SM. Tracking of activity and sedentary behaviors in childhood: the Iowa Bone Development Study. Am J Prev Med 2005; 29: 171-78.

LACERDA, Rodrigo Cabral Lacerda e MELO Fábio Antônio Tenório de. PREVALENCIA DE SOBREPESO E OBESIDADE EM ESCOLARES DE UMA INSTITUIÇÃO DE ENSINO PÚBLICA. Educação Física em Revista ISSN: 1983-6643 Vol.5 №2 Mai/Jun/Jul/Ago - 2011

MAESTRI, M.; FIAMONCINI, R.L. Perfil antropométrico de crianças na idade de 8 à 10 anos. Revista digital. Buenos Aires - Aifio 11, n. 97, jun./2006.

Medeiros CMM et al. Nutritional status and habits of life in school children. Journal of Human Growth and Development 2011; 21(3): 789-797.

MEIRELLES, M. C.; GOMES, C. S. P. Efeitos agudos da atividade contra-resistência sobre o gasto energético: revisitando o impacto das principais variáveis. Revista Brasileira de Medicina do Esporte, Niterói, v.10, n.2, p.122-30, 2004. http://dx.doi.org/10.1590/S1517- 86922004000200006

MELLO, E.; LUFT, V.; MEYER, F. Obesidade infantil: como podemos ser eficazes?.Jornal de Pediatria. 2004; 80(3) p.173-182. 
MELO et al. Anne Dal M. Prevalência de sobrepeso e obesidade em crianças de seis a dez anos de escolas municipais de área urbana. Rev Paul Pediatr 2010;28(1):48-54.

MONDINI et al. SOBREPESO E FATORES ASSOCIADOS EM CRIANÇAS INGRESSANTES NO ENSINO FUNDAMENTAL. Cad. Saúde Pública, Rio de Janeiro, 23(8):1825-1834, ago, 2007

Netto-Oliveira et al. Sobrepeso e obesidade em crianças. Rev Bras Cineantropom Desempenho Hum 2010, 12(2):83-89

OPAS. Doenças crônico-degenerativas e obesidade: estratégia mundial sobre alimentação saudável, atividade física e saúde. Organização Pan-Americana da Saúde,

Brasília, 2003.

RICARDO et al. Prevalência de sobrepeso e obesidade e indicadores de adiposidade central em escolares de Santa Catarina, Brasil Rev Bras Epidemiol 2009; 12(3): 424-35

RONQUE, et al. Prevalência de sobrepeso e obesidade em escolares de alto nível sócio-econômico em Londrina, Paraná, Brasil = Prevalence of overweight and obesity in schoolchildren of high socioeconomic level in Londrina, Paraná, Brazil. Revista de Nutrição, Campinas, SP, v.18, n.6, p.709-17, 2005. http://dx.doi.org/10.1590/S1415-52732005000600001

SOAR, C; VASCONCELOS, F. A. G; ASSIS, M. A. A. A relação cintura quadril e o perímetro da cintura associados ao índice de massa corporal em estudo com escolares.

Cadernos de Saúde Pública. 2004; 20(6):1609-1616.

SOCIEDADE BRASILEIRA DE PEDIATRIA. Departamento de Nutrologia. Obesidade na infância e adolescência - Manual de Orientação. São Paulo, 2008. 116 p. ISBN - 978-85-88520-05-9

SOTELO. Yêda de Oliveira Marcondes; COLUGNATI. Fernando A. B; TADDEI, José Augusto de Aguiar Carrazedo.Prevalências de Sobrepeso e obesidade entre Escolares da Rede Publica Segundo Três Critérios de Diagnóstico Antropométrico. Caderno de Saúde Pública, Rio de Janeiro, v. 20, n. 1, p.233, jan-fev 2004.

SILVA, Carolina Penteado Guerra . Prevalência de sobrepeso e obesidade em escolares do município de Fernandópolis - São Paulo. Revista Ciência \& Saúde, São Paulo, v.3,p13,out/Nov. 2005.

WHO. Organização Mundial da Saúde. The WHO child growth standards. 2006. Disponível em:http://www.who.int/childgrowth/en. Acesso em: 25 de setembro de 2013. 